\title{
Recurrent Monomorphic Post-Transplant Lymphoproliferative Disorder
}

National Cancer Institute

\section{Source}

National Cancer Institute. Recurrent Monomorphic Post-Transplant Lymphoproliferative Disorder. NCI Thesaurus. Code C157474.

The reemergence of monomorphic post-transplant lymphoproliferative disorder after a period of remission. 